\title{
XMM-Newton observation of the deep minimum state of PG 2112+059
}

\section{A spectrum dominated by reflection from the accretion disk?}

\author{
N. Schartel ${ }^{1}$, P. M. Rodríguez-Pascual ${ }^{1}$, M. Santos-Lleó ${ }^{1}$, L. Ballo $^{1}$, J. Clavel $^{2}$, M. Guainazzi ${ }^{1}$, \\ E. Jiménez-Bailón ${ }^{3,4}$, and E. Piconcelli ${ }^{5}$
}

\begin{abstract}
1 XMM-Newton Science Operations Centre, ESA, Villafranca del Castillo, Apartado 78, 28691 Villanueva de la Cañada, Spain e-mail: Norbert. Schartel@sciops. esa.int

2 Astrophysics Mission Division, ESA, SCT-SA, ESTEC, Postbus 299, 2200 AG Noordwijk, The Netherlands

3 Instituto de Astronomía, Universidad Nacional Autónoma de México, Apartado Postal 70-264, 04510 Mexico DF, México

4 LAEFF-INTA, Apdo 50727, 28080 Madrid, Spain

5 Osservatorio Astronomico di Roma (INAF), via Frascati 33, 00040 Monteporzio Catone, Italy
\end{abstract}

Received 8 May 2007 / Accepted 6 August 2007

\begin{abstract}
Context. Highly ionised absorbers and the frequent occurrence of relativistically broad iron fluorescence lines characterize the $0.2-10 \mathrm{keV}$ spectra of (soft) X-ray weak quasars.

Aims. We constrain the physical conditions of the absorber and the broad iron line of the X-ray weak quasar PG $2112+059$ in greater detail than in previous studies.

Methods. We analyse a 75 ks XMM-Newton observation of PG $2112+059$ performed in November 2005 and compare it with a 15 ks XMM-Newton observation taken in May 2003.

Results. PG $2112+059$ was found in a deep minimum state as its $0.2-12 \mathrm{keV}$ flux decreased by a factor of 10 in comparison to the May 2003 observation. During the deep minimum state the spectra show strong emission in excess of the continuum in the 3-6 keV region. The excess emission corresponds to an $E W=26.1 \mathrm{keV}$ whereas its shape resembles that of heavily absorbed objects.

The spectra of both observations of PG $2112+059$ can be explained statistically by a combination of two absorbers where one shows a high column density, $N_{\mathrm{H}} \sim 4.5 \times 10^{23} \mathrm{~cm}^{-2}$, and the other high ionisation parameters. As the ionisation parameter of the high flux state, $\xi \sim 34 \mathrm{erg} \mathrm{cm} \mathrm{s}^{-1}$, is lower than the value found for the deep minimum state, $\xi \sim 110 \mathrm{erg} \mathrm{cm} \mathrm{s}^{-1}$, either the absorbers are physically different or the absorbing material is moving with respect to the $\mathrm{X}$-ray source.

The spectra can also be explained by a continuum plus X-ray ionised reflection on the accretion disk, seen behind a warm absorber. The ionisation parameter of the high state $\left(\xi \sim 5.6 \mathrm{erg} \mathrm{cm} \mathrm{s}^{-1}\right)$ is higher than the ionisation parameter of the deep minimum state $\left(\xi \sim 0.2 \mathrm{erg} \mathrm{cm} \mathrm{s}^{-1}\right.$ ), as expected for a stationary absorber. The values found for the ionisation parameters are in the range typical for AGNs. The spectra observed during the deep minimum state are reflection dominated and show no continuum emission. These can be understood in the context of light bending near the supermassive black hole as predicted by Minutti and Fabian.

Conclusions. Light bending offers an alternative explanation for X-ray weak quasars and might challenge the suggestion that absorption is the primary cause of their X-ray weakness. If on a class level the weakness of X-ray weak quasars is caused by light bending then they offer unique possibilities to observe accretion disks near the supermassive black hole and even to test general relativity in the strong field.
\end{abstract}

Key words. quasars: general - quasars indiviudal: PG 2112+059 - accretion, accretion disks - black hole physics

\section{Introduction}

About $10 \%$ of quasars show an X-ray emission lower, by a factor of 10-30, than expected based on their luminosity in other energy bands (Laor et al. 1997; Wang et al. 1996; Elvis \& Fabbiano 1984). These quasars are called X-ray weak or soft X-ray weak quasars.

Brandt et al. (2000) showed that the optical to X-ray spectral index, $\alpha_{\mathrm{OX}}=0.372 \log \left(f_{2 \mathrm{keV}} / f_{3000} \AA\right)$ where $f_{2 \mathrm{keV}}$ and $f_{3000} \AA$ are flux densities, well separates soft X-ray weak from "normal" quasars, where soft X-ray weak quasars fulfil $\alpha_{\text {OX }} \leq-2$. $\mathrm{X}$-ray weak quasars show a strong correlation between $\alpha_{\text {OX }}$ and the CIV absorption equivalent width, which suggests that absorption is the primary cause of their soft X-ray weakness. The observed correlation between soft X-ray weakness and
C IV absorption equivalent width is in general agreement with models, which invoke a connection between orientation and absorption strength. With increasing inclination angle we may observe quasars, non-BAL X-ray weak quasars, BAL quasars and type 2 quasars. Although the explanation of X-ray weakness as being due to absorption is plausible, Brandt et al. (2000) noted that a uniform screen of absorbing material covering both the $\mathrm{X}$-ray source and the ultraviolet emission cannot reproduce the shape of the found correlation. Either the UV and X-ray absorbers are distinct or the X-ray weakness is due to a peculiar property of the nuclear emission, such as extreme variability or unusual steepness.

Brandt et al. (2000) found several notable differences between X-ray weak and "normal" quasars in the Boroson \& Green (1992) sample. Soft X-ray weak quasars are systematically 
characterised by low [O III] luminosities and equivalent widths as well as distinctive $\mathrm{H} \beta$ profiles. Most of them are located toward the weak [O III] end of the Boroson and Green eigenvector 1 , as are many BAL quasars. It has been suggested that unabsorbed Seyfert galaxies and quasars with eigenvectors $1 \mathrm{~s}$ similar to that found for X-ray weak quasars are characterized by extreme physical parameters in the nuclear region, i.e. a high mass accretion rate relative to the Eddington limit $\left(\dot{M} / M_{\text {Edd }}\right)$.

The X-ray weak broad absorption line quasar PG 2112+059 shows a complex spectrum as well as a remarkable variability in the $0.2-10 \mathrm{keV}$ region (Gallagher et al. 2004; Schartel et al. 2005). We use a deep XMM-Newton observation with a combination of spectral information and variability to constrain the physical properties of this quasar. In this paper we present the analysis of the new XMM-Newton data from 2005. We compare the results with previous X-ray observations of PG 2112+059 and the XMM-Newton observation in 2002. The paper is organized as follows: in Sect. 2 we provide a summary of the previous X-ray observations of PG 2112+059. Both XMM-Newton observations and the performed analysis are described in Sect. 3. The observed variability is presented in Sect. 4 and the spectral analysis in Sect. 5. We discuss our results in Sect. 6 and draw some conclusions in Sect. 7.

\section{2. $P G ~ 2112+059$}

PG $2112+059$ is a member of the Palomar bright quasar survey (Schmidt \& Green 1983) and is located at a redshift of $z=0.456$ (Véron-Cetty \& Véron 2000).

The Hubble Space Telescope spectrum of PG 2112+059 (Jannuzi et al. 1998) shows broad CIV absorption lines (Gallagher et al. 2001) which classifies the source as a Broad Absorption Line (BAL) quasar. The quasar was observed with ROSAT in 1991 and based on the data, Wang (1996) determined a low optical to X-ray spectral index characteristic of X-ray weak quasars.

ASCA observed PG 2112+059 in October 1999 (Gallagher et al. 2001) and in September 2002 the quasar was the target of a Chandra observation (Gallagher et al. 2004). The observations revealed a dramatic variability, where the ASCA observation showed the highest flux, which was approximately a factor of four higher than both in the earlier ROSAT observation and in the later Chandra observation. An intermediate continuum flux state was measured with XMM-Newton in May 2003 (Schartel et al. 2005).

The spectrum of PG 2112+059 taken in October 1999 with ASCA showed clear indication of absorption but the statistics were insufficient to discriminate between a neutral absorber, partial covering by a neutral absorber or an ionised absorber. Neutral absorption could be excluded for PG $2112+059$ for the Chandra spectrum taken in September 2002. An ionised absorber as well as a partially covering neutral absorber are able to describe the Chandra data statistically (Gallagher et al. 2004). Both absorption scenarios require an increase of the absorbing column density between 1999 and 2002. On the other hand the XMM-Newton spectra of PG 2112+059 from May 2003 require an ionised absorber. A neutral absorber as well as a partial covering absorption scenario can be excluded from a statistical point of view (Schartel et al. 2005).

The October 1999 ASCA spectra of PG 2112+059 showed no evidence for iron line emission and Gallagher et al. (2001) determined an upper limit for the narrow neutral iron line equivalent width of less than $210 \mathrm{eV}$. Surprisingly, the spectrum of the quasar taken with Chandra in September 2002 shows a broad
Table 1. Exposure details of the November 2005 observation of PG 2112+059.

\begin{tabular}{rcccc}
\hline \hline N. $^{1}$ & In. $^{2}$ & Filter & $\begin{array}{c}\text { Start } \\
\text { day and time } \\
{[\mathrm{UT}]}\end{array}$ & $\begin{array}{c}\text { Duration } \\
{[\mathrm{ks}]}\end{array}$ \\
\hline 1 & M1 & thin 1 & 20 at $21: 13: 12$ & 75.9 \\
2 & M2 & thick & 20 at $21: 13: 12$ & 75.9 \\
3 & pn & thin 1 & 20 at $21: 35: 54$ & 74.2 \\
6 & OM & $V$ & 20 at $21: 17: 50$ & $3 \times 1.9$ \\
9 & OM & $U$-NoBar & 20 at $23: 08: 11$ & $3 \times 1.9$ \\
12 & OM & $B$ & 21 at 00:58:32 & $3 \times 1.9$ \\
15 & OM & WHITE & 21 at 02:48:53 & $3 \times 1.9$ \\
18 & OM & VISIBLE GRISM 2 & 21 at 04:39:14 & $3 \times 4.0$ \\
21 & OM & UVW1 & 21 at 08:44:35 & $3 \times 1.9$ \\
24 & OM & UVM2 & 21 at 11:04:56 & $3 \times 1.9$ \\
27 & OM & UVW2 & 21 at $12: 55: 17$ & $3 \times 1.9$ \\
30 & OM & UV GRISM 1 & 21 at $14: 45: 38$ & $3 \times 4.0$ \\
\hline
\end{tabular}

${ }^{1}$ Exposure identifier; OM performed three exposures with each filter. Only for the first exposure is the exposure identifier provided. ${ }^{2}$ instrument, where M1 stands for MOS 1 and M2 for MOS2.

iron line (Gallagher et al. 2004). Such a broad line with the same width and the same flux is below the detection limit of the ASCA spectrum from 1999 due to the much higher continuum flux. Although the XMM-Newton spectra from May 2003 do not require a broad iron line for a satisfying statistical description, adding a line with the same width and flux as detected in the spectrum taken with Chandra in September 2002 improves the description of the data at the $97 \%$ probability level (Schartel et al. 2005). The XMM-Newton spectra can be understood provided that the continuum is increased with respect to the Chandra observation; the broad iron line emission is constant.

\section{Observations and data reduction}

In 2005 XMM-Newton (Jansen et al. 2001) observed PG 2112+059 for about $76 \mathrm{ks}$. The observation started at $21 \mathrm{~h} 12 \mathrm{~min}$ (UT) on the 20th of November 2005 and is archived under the observation identifier 0300310201 in the XMM-Newton scientific archive. In order to take advantage of the latest calibration progress and to allow an optimal comparison between the two XMM-Newton observations we have reanalysed the XMM-Newton data taken in May 2003. Details of this observation are provided in Schartel et al. (2005). This observation is archived under the observation identifier 0150610201.

During the XMM-Newton pointings three scientific instruments simultaneously observe: the Reflection Grating Spectrometer (RGS; Brinkman et al. 2001), the European Photon Imaging Camera (EPIC) and the Optical Monitor (OM; Mason et al. 2001). EPIC consists of three CCD cameras: the pn-camera (Strüder et al. 2001) and two MOS-cameras (Turner et al. 2001). All EPIC exposures of PG $2112+059$ were taken in the Full Frame mode with different optical blocking filters in the light path. The details are provided in Table 1. OM performed in total 28 observations with different filters or grisms in the optical light path. All OM exposures were performed in the "Science User Defined" image mode. The details are provided in Table 1.

We processed the data with the XMM-Newton Science Analysis System (SAS) v. 7.0 (linux; compare Loiseau et al. 2006) with the calibrations from November 2006.

The pipeline products provided by the Survey Science Centre (Watson et al. 2001) indicated that the RGS spectra of 
Table 2. Screening for low background level, source position and background extraction area.

\begin{tabular}{|c|c|c|c|c|c|c|c|c|c|c|c|}
\hline \multirow[t]{2}{*}{ N. ${ }^{1}$} & \multirow[t]{2}{*}{ C. ${ }^{2}$} & \multirow{2}{*}{$\begin{array}{c}\text { Source position } \\
\mathrm{RA}^{4} \\
{[\mathrm{~h}: \mathrm{m}: \mathrm{s}]}\end{array}$} & \multirow{2}{*}{$\begin{array}{c}\operatorname{Dec}^{4} \\
{[\mathrm{~d}: \mathrm{m}: \mathrm{s}]}\end{array}$} & \multicolumn{3}{|c|}{ Screening parameters ${ }^{3}$} & \multicolumn{2}{|c|}{ Background extraction area } & \multicolumn{3}{|c|}{ Net source } \\
\hline & & & & $\begin{array}{c}\text { Energy }^{5} \\
{[\mathrm{keV}]}\end{array}$ & $\begin{array}{l}r^{6} \\
{\left[{ }^{\prime}\right]}\end{array}$ & $\begin{array}{l}\mathrm{CR}^{7} \\
{\left[\mathrm{~s}^{-1}\right]}\end{array}$ & $\begin{array}{c}\mathrm{RA}^{4} \\
{[\mathrm{~h}: \mathrm{m}: \mathrm{s}]}\end{array}$ & $\begin{array}{l}\mathrm{Dec}^{4} \\
{[\mathrm{~d}: \mathrm{m}: \mathrm{s}]}\end{array}$ & $\begin{array}{l}r^{8} \\
{\left[{ }^{\prime \prime}\right]}\end{array}$ & $\begin{array}{r}\mathrm{CR}^{(9)} \\
{\left[10^{-2} \mathrm{~s}^{-1}\right]}\end{array}$ & $\begin{array}{r}T^{10} \\
{[\mathrm{ks}]}\end{array}$ \\
\hline \multicolumn{12}{|c|}{ Observation from 14.5.2003 / observation identifier 0150610201} \\
\hline 1 & pn & 21:14:52.47 & $6: 07: 42.0$ & $0.2-12.0$ & $1-11$ & 10. & $21: 14: 48.393$ & $6: 07: 20.75$ & 30 & $11.95 \pm 0.42$ & 7.1 \\
\hline 2 & M1 & $21: 14: 52.44$ & $6: 07: 41.5$ & $0.2-10.0$ & $1-14$ & 5.0 & source & source & $40-105$ & $3.50 \pm 0.18$ & 10.8 \\
\hline 3 & M2 & $21: 14: 52.44$ & $6: 07: 41.5$ & $0.2-10.0$ & $1-14$ & 5.0 & source & source & $40-105$ & $3.59 \pm 0.18$ & 10.8 \\
\hline \multicolumn{12}{|c|}{ Observation from 20.10.2005 / observation identifier 0300310201} \\
\hline 1 & M1 & $21: 14: 52.60$ & $6: 07: 41.0$ & $0.2-10.0$ & $1-14$ & 2.0 & source & source & $40-105$ & $0.37 \pm 0.02$ & 72.3 \\
\hline 2 & M2 & $21: 14: 52.60$ & 6:07:41.0 & $0.2-10.0$ & $1-14$ & 2.0 & source & source & $40-105$ & $0.33 \pm 0.02$ & 73.6 \\
\hline 3 & pn & 21:14:52.55 & $6: 07: 42.0$ & $0.2-12.0$ & $1-11$ & 4.0 & 21:14:48.769 & 6:07:21.99 & 30 & $1.19 \pm 0.05$ & 62.5 \\
\hline
\end{tabular}

${ }^{1}$ Exposure identifier; ${ }^{2}$ EPIC camera where M1 stands for MOS1 and M2 for MOS2; ${ }^{3}$ for the screening the data were binned with 100 s; ${ }^{4}$ in J2000; ${ }^{5}$ energy range considered for the screening; ${ }^{6}$ the inner and the outer radius of the screening annulus; ${ }^{7}$ count rate threshold for rejection of screening; ${ }^{8}$ radius of background region (for annular background areas the inner and the outer radius are provided); ${ }^{9}$ background corrected source count rate in the energy range of $0.2-12.0 \mathrm{keV}$ for pn and $0.2-10.0 \mathrm{keV}$ for MOS, respectively; ${ }^{10}$ accumulated exposure time.

PG $2112+059$ are either extremely weak or that the source is not detected at all with RGS. We inspected the processed RGS events in the dispersion-cross-dispersion-plane without finding any trace of a source spectrum for either of the two XMM-Newton observations.

We processed the pn and MOS data with the EPPROC and EMCHAIN routines of SAS, respectively. For pn single and double events were considered, which are characterized by pattern 0 to 4 . For MOS observations, events with pattern 0-12 were chosen, which correspond to single, double, triple and quadruple events. The applied pattern selection follows the recommendation (Kirsch et al. 2006), ensures an optimal energy calibration and allows standard detector response and effective area calculation.

We followed the procedure of Piconcelli et al. (2005) for screening for time ranges with low radiation background level. The source extraction areas, the energy ranges used for the screening, the corresponding rejection thresholds, the screening background extraction areas and the final accumulated exposure times for each EPIC exposure are given in Table 2.

The source events were extracted from a circular region with $r=15^{\prime \prime}$ centred at the peak of the emission, which was determined by eye. Table 2 gives the source position used for the extraction. The background was estimated from source-free regions near the source. For the pn-camera an offset circular region was chosen and for the MOS-cameras an annulus around the source extraction region was selected. The background for each exposure was determined from counts collected at the same CCD in order to avoid the propagation of calibration differences. The centre of the circular background extraction region of the pn data was selected to have CCD $y$-coordinates similar to the source extraction region, which minimizes effects resulting from the charge transfer efficiency dependency on the detector coordinates. All areas are specified in Table 2. The detector response and the effective area were generated with the SAS routines RMFGEN and ARFGEN, respectively.

We processed the OM observations with the OMGCHAIN and OMICHAIN routine of SAS, which applies all required astrometric and photometric corrections. The two OM grisms were used in both observations of PG $2112+059$. In both observation the UV flux of PG $2112+059$ was too low to accumulate useful spectra. The spectra obtained with the visible grism in the light path of OM are of sufficient quality. The long observation of PG $2112+059$ allowed in addition OM exposures with
Table 3. Fluxes through OM filters.

\begin{tabular}{lcc}
\hline \hline Filter & $\begin{array}{c}\text { Flux } \\
(1)\end{array}$ & $\begin{array}{c}\text { Ratio } \\
(2)\end{array}$ \\
\hline$V$ & $2.08 \pm 0.01$ & $1.01 \pm 0.02$ \\
$B$ & $2.91 \pm 0.04$ & $1.03 \pm 0.01$ \\
$U$ & $3.83 \pm 0.01$ & $1.00 \pm 0.01$ \\
$U V W 1$ & $4.37 \pm 0.07$ & $1.03 \pm 0.02$ \\
$U V M 2$ & $4.76 \pm 0.06$ & $1.03 \pm 0.04$ \\
$U V W 2$ & $6.28 \pm 0.03$ & $1.01 \pm 0.07$ \\
\hline
\end{tabular}

(1): Flux in units of $10^{-15} \mathrm{erg} / \mathrm{cm}^{2} / \mathrm{s} / \AA ̊ \AA$; (2): Maximum to minimum flux ratio.

the $U, V, B, U V W 1, U V M 2$ and $U V W 2$ filters in the light path. For each filter three images were obtained. We determined the fluxes through the mean of the three values and provide the standard derivation as an estimate of their error. The obtained values are provided in Table 3.

\section{Timing analysis}

\section{1. $O M$}

The sequence of OM exposures in November 2005 allows us to set upper limits to the variability of the source in different spectral bands on timescales of a few hours. The average and the ratio of the maximum to the minimum flux are given in Table 3 for each filter and show that the flux remains constant in all optical and UV broad-band filters within a few percent.

Only OM Grism exposures were performed in the XMM observation on May 2003. Since PG $2112+059$ is rather faint in the UV for the OM UV-Grism, we can only compare the optical spectra obtained with the $V$-Grism. The optical spectrum obtained in November 2005 is systematically fainter than the optical spectrum in May 2003 by $\sim 10 \%$. This difference is only slightly larger than the measurement errors: $\sim 5 \%$.

\subsection{X-ray}

We based the X-ray variability analysis upon the pn observation as this camera has the largest effective area of all X-ray instruments onboard XMM-Newton. PG 2112+059 shows a dramatic decrease of the X-ray flux between May 2003 and November 2005: the $0.2-12 \mathrm{keV}$ net source count rate dropped 
by a factor of 10 from $0.116 \pm 0.004 \mathrm{~s}^{-1}$ to $0.0117 \pm 0.0004 \mathrm{~s}^{-1}$. The net source count rate is determined from the screened data. As the source shows in the November 2005 observation the lowest flux of all X-ray observations reported so far, we refer in the following to it as the "deep minimum state" of the source.

The short time variability within the observation was tested based on the events of the unscreened data. The source and background region were defined as described in Sect. 3. We generated source light curves with a binning time of $10000 \mathrm{~s}$ and $5000 \mathrm{~s}$ for the $350 \mathrm{eV}-500 \mathrm{eV}, 500 \mathrm{eV}-2 \mathrm{keV}, 2 \mathrm{keV}-12 \mathrm{keV}$ and $300 \mathrm{eV}-12 \mathrm{keV}$ energy regions, respectively. We were not able to identify any time bin showing a significant derivation from the mean value. Based on this finding in combination with the size of the error bars, we estimate that the source variability is below $20 \%$ during the November 2005 observation.

\section{Spectral analysis}

We performed the spectral analysis of the MOS spectra over the energy range from $0.2 \mathrm{keV}$ to $10.0 \mathrm{keV}$ and of the pn spectra over the 0.2 to $12.0 \mathrm{keV}$ energy range, following the current recommendations (Kirsch 2006).

In order to take full advantage of the progress in calibration and, especially, to allow a rigorous comparison, we provide in the following the spectral modelling for the deep minimum state from November 2005 as well as of the high state from May 2003. In both cases we have added the two MOS spectra and have calculated the corresponding effective areas and response matrices. We have done this for the November 2005 observation although the two MOSs were exposed with different optical blocking filters in the light path. However, the accumulated counts at low energies are too few to take advantage of the differences in effective area as originally intended with the selection of the different optical blocking filters.

The pn and (added) MOS spectra were binned with a signalto-noise ratio of $>5$ restricted to a maximum of three bins per spectral resolution element. With this choice for the binning, over-sampling is excluded, a Gaussian distribution is approximately reached and $\chi^{2}$ statistics can be applied in the special fitting. The spectral analysis was performed with XSPEC 12.3.0c (Linux version, Arnaud 2006). This software package uses the modified minimum $\chi^{2}$ method (Kendall \& Stuart 1973) for estimation of the spectral parameters, which requires applied binning. The errors of estimated parameters are given for the $90 \%$ confidence region for a single interesting parameter $\left(\Delta \chi^{2}=2.7\right.$, Avni 1976).

For all spectral models we assumed Galactic foreground absorption. The Galactic equivalent column density in the direction of the quasar was determined through pointed $21 \mathrm{~cm}$ radio observations to be $N_{\mathrm{H}}=6.26 \times 10^{20} \mathrm{~cm}^{-2}$ with an error of $5 \%$ (Lockman \& Savage 1995). For the different models the best estimated parameters, their errors and the statistical parameter are provided in Table 4; the same quantities for the emission or absorption components of the models are reported in Table 5.

The spectral shape, observed during the deep minimum state of PG 2112+059, differs significantly from the shape observed during the high state in May 2003. In the 3-6 keV region the minimum state shows a bump, like sources that are partially covered by material with a high column density. Figure 1 shows the ratio between the EPIC data of the deep minimum state and a hypothetical canonical power law spectrum with a photon index of $\Gamma=2$ absorbed by material of Galactic column density. This bump was not present in the spectrum taken during the high state of the source. Given this appearing spectral feature we first tried to explain the spectra in terms of absorption and changes of it.

\subsection{Continuum modeling with absorbers}

Assuming a power law continuum we can exclude a neutral absorber $\left(\chi^{2}=108.3\right.$, d.o.f. $\left.=51\right)$ as well as a neutral absorber that partially covers the source $\left(\chi^{2}=68.3\right.$, d.o.f. $\left.=50\right)$ for the high state of the source, whereas an ionised absorber allows a satisfying description of the spectra (Table 4, fit 1). For the deep minimum state a neutral absorber $\left(\chi^{2}=204.9\right.$, d.o.f. $\left.=35\right)$ as well as ionised absorber $\left(\chi^{2}=76.1\right.$, d.o.f. $\left.=34\right)$ can be excluded, whereas a neutral absorber partially covering the source provides a possible description of the source spectra (fit 8). As an ionised absorber cannot describe the deep minimum state of the source, we can exclude that a change of the ionisation state of the absorbing material in response to source variability explains the spectral differences.

In order to test for more complex absorption scenarios, we tried first a model assuming that part of the light is absorbed by neutral material and that the remaining part of the light is absorbed by ionised material. This double-absorber scenario provides a statistically significant description of the spectra obtained during the high state (fit 2) as well as during the deep minimum state of the source (fit 9). During the high state the covering fraction is $54 \%$ for the neutral material $\left(N_{\mathrm{H}}=29_{-13}^{+18} \times 10^{22} \mathrm{~cm}^{-2}\right)$ and $46 \%$ for the ionised material $\left(N_{\mathrm{H}}=3.2_{-1.2}^{+1.0} \times 10^{22} \mathrm{~cm}^{-2}\right)$. The corresponding numbers for the low state are $94 \%\left(N_{\mathrm{H}}=\right.$ $\left.46_{-7}^{+7} \times 10^{22} \mathrm{~cm}^{-2}\right)$ and $6 \%\left(N_{\mathrm{H}}=2.1_{-1.6}^{+0.9} \times 10^{22} \mathrm{~cm}^{-2}\right)$. The absorption scenario even allows a description of both states assuming constant column densities but allowing different ionisation states and relative normalization of the continuum components (fit 20), where the latter equals a change of the covering factors. The obtained high state best-fit values correspond to a covering fraction of $61 \%$ for the neutral absorber $\left(N_{\mathrm{H}}=42_{-5}^{+9} \times 10^{22} \mathrm{~cm}^{-2}\right)$ and $39 \%$ for the ionised material $\left(N_{\mathrm{H}}=3.01_{-0.9}^{+0.5} \times 10^{22} \mathrm{~cm}^{-2}\right)$. The joint fit of both states estimates the flux of the high state to be 3.8 times the flux emitted during the deep minimum state. Hence assuming constant location and density of the absorbing material we expect the ionisation parameter of the high state to be 3.8 times higher than the ionisation parameter of the deep minimum state. This is in sharp contrast to the observations, which show instead a high state ionisation parameter 3 times lower than the low state one.

Similarly we tested a scenario with an ionised absorber and a neutral absorber that partially covers the source in the light path to the X-ray source. This scenario implies a layer of material that is located sufficiently near to the continuum source to be ionised and clouds of neutral material. This scenario provides an acceptable statistical description of the spectra taken during both states of the source (fit 3 and fit 10). Again the scenario provides a statistically acceptable description of both states assuming constant column densities. However, it requires a change of the covering factor of the neutral absorber beside the change of the ionisation states (fit 21). Similar to the previous scenario we can compare the variability with the change of the ionisation parameter. Assuming a constant location and density of the absorbing material we would expect that a flux variability of a factor of $\sim 3$ implies the ionisation parameter of the high state to be a factor of 3 higher than the ionisation parameter of the deep minimum state. Again the opposite is the case: the ionisation parameter in the high state is a factor of 3 lower than the low state. 
Table 4. Spectral fits of the EPIC spectra of PG $2112+059$.

\begin{tabular}{|c|c|c|c|c|c|c|c|c|c|c|}
\hline \multirow[t]{3}{*}{ 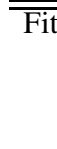 } & \multirow[t]{3}{*}{$\overline{\text { it } \text { Model }^{a}}$} & \multirow{2}{*}{\multicolumn{4}{|c|}{ Absorber }} & \multirow{2}{*}{\multicolumn{2}{|c|}{$\begin{array}{l}\text { Continuum I } \\
\text { power-law }\end{array}$}} & \multirow{3}{*}{$\begin{array}{c}\text { Continuum II } \\
\text { power-law } \\
N^{e} \\
{\left[\mathrm{keV}^{-1} \mathrm{~cm}^{-2} \mathrm{~s}^{-1}\right]}\end{array}$} & \multicolumn{2}{|c|}{ Statistic } \\
\hline & & & & & & & & & & \\
\hline & & \multirow[t]{2}{*}{$\begin{array}{c}N_{\mathrm{H}}^{b} \\
{\left[10^{22} \mathrm{~cm}^{-2}\right]}\end{array}$} & $c f^{c}$ & $\begin{array}{c}N_{\mathrm{H}}^{b} \\
{\left[10^{22} \mathrm{~cm}^{-2}\right]}\end{array}$ & $\begin{array}{c}\xi^{d} \\
{\left[\mathrm{erg} \mathrm{cm} \mathrm{s}^{-1} \text { ] }\right.}\end{array}$ & $\Gamma$ & \multirow[t]{2}{*}{$\begin{array}{c}N^{e} \\
{\left[\mathrm{keV}^{-1} \mathrm{~cm}^{-2} \mathrm{~s}^{-1}\right]}\end{array}$} & & $\chi^{2}$ & $d^{g}$ \\
\hline \multicolumn{9}{|c|}{ High state / observation from May 2003} & & \\
\hline 1 & $i a * p$ & & & $6.7_{-1.9}^{+2.3}$ & $87_{-28}^{+24}$ & $1.82_{-0.08}^{+0.14}$ & $1.2_{-01}^{+0.2} \times 10^{-4}$ & & 44.7 & 50 \\
\hline 2 & $n a * p+i a * p$ & $29_{-13}^{+18}$ & - & $3.2_{-12}^{+1.0}$ & $39_{-16}^{+283}$ & $2.19_{-0.22}^{+0.12}$ & $1.4_{-0.9}^{+0.1} \times 10^{-4}$ & $1.2_{-02}^{+0.1} \times 10^{-4}$ & 35.4 & 48 \\
\hline 3 & $p a * i a * p$ & $28_{-13}^{+30}$ & $0.51_{-0.20}^{+0.14}$ & $3.3_{-12}^{+1.5}$ & $39_{-17}^{+102}$ & $2.14_{-0.16}^{+0.16}$ & $2.4_{-07}^{+0.9} \times 10^{-4}$ & & 35.1 & 48 \\
\hline 4 & $i a *(p+i a * p)$ & & & $3.3_{-1.7}^{+1.5}$ & $39_{+22}^{-17}$ & $2.14_{-0.17}^{+0.18}$ & $1.2_{-0.4}^{+0.4} \times 10^{-4}$ & & & \\
\hline & & & & $35_{-20}^{+65}$ & $2.4_{+4900}^{-2.4}$ & & $1.2_{-1.0}^{+0.4} \times 10^{-4}$ & & 34.9 & 47 \\
\hline 5 & $(p a * i a * p)+g a$ & $26_{-13}^{+30}$ & $0.49_{-0.23}^{+0.14}$ & $3.3_{-1.2}^{+1.5}$ & $39_{-17}^{+22}$ & $2.14_{-0.17}^{+0.16}$ & $2.3_{-0.7}^{+1.0} \times 10^{-4}$ & & 34.2 & 47 \\
\hline 6 & $i a *(p+b l(r e))$ & & & $3.1_{-1.5}^{+1.6}$ & $35_{-22}^{+18}$ & $1.90_{-0.10}^{+0.11}$ & $9.9_{-1.2}^{+1.8} \times 10^{-5}$ & & 35.4 & 45 \\
\hline 7 & $i a *(p+b l(r e))$ & & & $2.9_{-1.4}^{+1.9}$ & $34_{-22}^{+32}$ & $1.90_{-0.37}^{+0.18}$ & $9.7_{-2.3}^{+2.1} \times 10^{-5}$ & & 36.0 & 46 \\
\hline \multicolumn{11}{|c|}{ Deep minimum state / observation from November 2005} \\
\hline 8 & $p a * p$ & $40_{-5}^{+6}$ & $0.96_{-0.17}^{+0.14}$ & & & $2.23_{-0.07}^{+0.07}$ & $9.1_{-2.9}^{+0.7} \times 10^{-5}$ & & 46.6 & 34 \\
\hline 9 & $n a * p+i a * p$ & $46_{-7}^{+7}$ & - & $2.1_{-16}^{+0.9}$ & $74_{-53}^{85}$ & $2.28_{-0.013}^{+0.07}$ & $1.0_{-0.4}^{+0.9} \times 10^{-4}$ & $5.9_{-12}^{+1.1} \times 10^{-6}$ & 40.4 & 32 \\
\hline 10 & $p a * i a * p$ & $44_{-7}^{+-8}$ & $0.94_{-0.03}^{+0.02}$ & $2.2_{-16}^{+2.6}$ & $80_{-66}^{+105}$ & $2.25_{-0.19}^{+0.13}$ & $9.8_{-3.1}^{+0.4} \times 10^{-5}$ & & 40.1 & 32 \\
\hline 11 & $i a *(p+i a * p)$ & & & $2.3_{-1.1}^{+3.6}$ & $\begin{array}{l}-66 \\
87_{+98}^{-70}\end{array}$ & $2.20_{-0.19}^{+0.20}$ & $5.7_{-13}^{+1.1} \times 10^{-6}$ & & & \\
\hline & & & & $98_{(-32}^{+2.8}$ & $91_{+62}^{+668}$ & & $9.2_{-3.4}^{+\frac{1}{5.3}} \times 10^{-5}$ & & 34.2 & 31 \\
\hline 12 & $(p a * i a * p)+g a$ & $42_{-7}^{+8}$ & $0.93_{-0.04}^{+0.02}$ & $2.4_{-1.8}^{+3.3}$ & $88_{-69}^{+98}$ & $2.21_{-0.19}^{+0.20}$ & $8.5_{-1.9}^{+1.9} \times 10^{-5}$ & & 33.5 & 31 \\
\hline $13^{\dagger}$ & $s w * p$ & $\left(\sigma=0.16_{-0.04}^{+0.34}\right.$, & $\left.z=0.34_{-0.09}^{+0.32}\right)$ & $4.0_{-4.1}^{+3.8}$ & $152_{-152}^{+150}$ & $1.87_{-0.11}^{+0.23}$ & $1.3_{-0.2}^{+0.9} \times 10^{-4}$ & & 42.6 & 48 \\
\hline $14^{\dagger}$ & $p a * e d * p$ & $50_{-9}^{+14^{-c}}$ & $0.97_{-0.07}^{+0.03}$ & & & $2.00^{f}$ & $9.0_{-1.6}^{+2.1} \times 10^{-5}$ & & 16.0 & 14 \\
\hline $15^{\dagger}$ & $p a * n o * p$ & $55_{-11}^{+24}$ & $0.95_{-0.09}^{+0.05}$ & & & $2.00^{f}$ & $9.4_{-1.8}^{+3.0} \times 10^{-5}$ & & 15.8 & 14 \\
\hline $16^{\dagger}$ & $s w * p$ & $\left(\sigma=0.00_{-0.00}^{+0.12}\right.$, & $\left.z=0.18_{-0.18}^{+0.04}\right)$ & $50_{-5}^{+0}$ & $330_{-100}^{+110}$ & $3.70_{-0.64}^{+1.59}$ & $1.3_{-1.3}^{+22} \times 10^{-3}$ & & 21.1 & 13 \\
\hline 17 & $n a *(p+l a)$ & $0.0_{-00}^{+2.8} \times 10^{-2}$ & & & & $1.97_{-0.21}^{+0.21}$ & $4.3_{-03}^{+0.4} \times 10^{-6}$ & & 50.5 & 30 \\
\hline 18 & $i a *(p+b l(r e))$ & & & $0.6_{-0.4}^{+0.3}$ & $0.01_{-0.01}^{+0.31}$ & $1.94_{-0.29}^{+0.22}$ & $0.0_{-0.0}^{+1.0} \times 10^{-6}$ & & 29.9 & 29 \\
\hline 19 & $i a *(p+b l(r e))$ & & & $0.7_{-0.5}^{+0.3}$ & $0.03_{-0.03}^{+0.36}$ & $1.87_{-0.22}^{+0.29}$ & $0.0^{+2.1} \times 10^{-6}$ & & 35.7 & 30 \\
\hline \multicolumn{11}{|c|}{ Both states $^{g}$ (high / deep minimum) } \\
\hline 20 & $n a * p+i a * p$ & $42_{-5}^{+9}$ & - & $3.01_{-0.9}^{+0.5}$ & $34_{-12}^{+18}$ & $2.24_{-0.19}^{+0.07}$ & $2.0_{-1.3}^{+0.6} \times 10^{-4}$ & $1.3_{-0.2}^{+0.1} \times 10^{-4}$ & & \\
\hline & & & & & $110_{-51}^{+12}$ & $2.20_{-0.16}^{+0.15}$ & $8.1_{-4.4}^{+3.3} \times 10^{-5}$ & $6.0_{-0.9}^{+1.2} \times 10^{-6}$ & 78.8 & 82 \\
\hline 21 & $p a * i a * p$ & $43_{-6}^{+6}$ & $0.58_{-0.10}^{+0.05}$ & $3.1_{-0.8}^{+0.6}$ & $34_{-11}^{+51}$ & $2.18_{-0.16}^{+0.07}$ & $2.9_{-0.4}^{+0.4} \times 10^{-4}$ & & & \\
\hline & & & $0.94_{-0.03}^{+0.01}$ & & $106_{-44}^{+90}$ & $2.27_{-0.21}^{+0.05}$ & $1.0_{-0.3}^{+0.4} \times 10^{-4}$ & & 77.9 & 82 \\
\hline 22 & $i a *(p+i a * p)$ & & & $4.3_{-1.1}^{+1.3}$ & $39_{+23}^{-16}$ & $1.93_{-0.10}^{+0.09}$ & $1.6_{-0.5}^{+0.4} \times 10^{-5}$ & & & \\
\hline & & & & $100_{-31}^{+0.0}$ & $\begin{array}{c}4430_{-1000}^{+570} \\
117^{-35}\end{array}$ & & $\begin{array}{c}1.4_{-0.3}^{+0.8} \times 10^{-4} \\
5.90^{+1.07} \times 10^{-6}\end{array}$ & & & \\
\hline & & & & & $80.3^{+27.5}$ & & -2.01 & & 76.8 & 82 \\
\hline 23 & $i a *(p+b l(r e))$ & & & $0.9_{-0.2}^{+0.3}$ & $\begin{array}{l}5.7_{-5.7}^{+6.2} \\
+3.2\end{array}$ & $1.81_{-0.10}^{+0.11}$ & $7.4_{-0.8}^{+0.9} \times 10^{-5}$ & & & \\
\hline & & & & & $0.2_{-0.17}^{+0.7}$ & & $0.0_{-0.0}^{+0.0} \times 10^{-7}$ & & 79.7 & 80 \\
\hline 24 & $i a *(p+b l(r e))$ & & & $0.9_{-0.3}^{+0.3}$ & $5.8_{-3.3}^{+1.1}$ & $1.80_{-0.09}^{+0.11}$ & $7.3_{-0.8}^{+0.9} \times 10^{-5}$ & & & \\
\hline & & & & & $0.2_{-0.17}^{+0.5}$ & & $0.0_{-0.0}^{+8.9} \times 10^{-7}$ & & 83.9 & 81 \\
\hline 25 & $i a *(p+b l(r e))$ & & & $0.9_{-0.2}^{+0.3}$ & $5.2_{-3.3}^{+9.6}$ & $1.79_{-0.16}^{+0.12}$ & $7.2_{-1.0}^{+1.0} \times 10^{-5}$ & & & \\
\hline & & & & & $0.2_{-0.2}^{+0.5}$ & & $0.0_{-0.0}^{+7.9} \times 10^{-7}$ & & 83.8 & 80 \\
\hline
\end{tabular}

The errors are provided for the $90 \%$ confidence level. Fits labeled with $\dagger$ were performed over the $2-10 \mathrm{keV}$ energy range for MOS and the 2-12 keV energy range of pn. Explanation of labels: ${ }^{a}$ explanation of the used models: $p$ : power-law (XSPEC: pow), na: neutral absorber at the redshift of the source (XSPEC: zphabs), pa: partial covering with neutral absorber at the redshift of the source (XSPEC: zpcfabs), ia: ionized absorption at the redshift of the source (XSPEC: absori), sw: partially ionised absorbing material with large velocity shear (XSPEC: swind1) $e d$ : absorption edge at the redshift of the source (XSPEC: zedge), no: notch line absorption (XSPEC: notch), ga: Gaussian line at the redshift of the source (XSPEC: zgauss), la: relativistic disk line after Laor (XSPEC: laor, Laor 1997), bl: relativistic blurring from an accretion disk around a rotating black hole (XSPEC: kdblur, modified from laor model by Fabian and Johnstone), re: reflection by a constant density illuminated atmosphere (XSPEC: Reflion, Ross \& Fabian 2005). Parameters: ${ }^{b}$ equivalent column density; ${ }^{c}$ covering fraction; ${ }^{d}$ ionization level of absorbing material $\xi=L / n r^{2}$, as defined in XSPEC; ${ }^{e}$ photon flux at $1 \mathrm{keV} ;{ }^{f}$ fixed; ${ }^{g}$ degrees of freedom. In all cases, where both states are fitted simultaneously, the best estimated parameters are provided in two lines: The first line provides the parameters for the high state and the second line for the deep minimum state. In the second line, only parameters are given which were free to vary with respect to the high state (first line).

Next we considered the possibility that PG $2112+059$ is covered by an ionised absorber partially covering the $\mathrm{X}$-ray source in combination with a further layer of ionised material that covers the X-ray source completely. This scenario allows a satisfying description of the two observations individually (fit 4 and 11) as well as of the two observations together (fit 22). In the latter case a high column density absorber covers about $90 \%$ of the $\mathrm{X}$-ray source and a low density absorber covers the entire source.
Again, it is interesting to check the behaviour of the ionization parameters of the two absorbers as the source flux changes. As before for the low-column-density absorber, the ionisation parameter in the high state is a factor of 3 lower than in the deep minimum state whereas we would have expected a drop by a factor of three. For the high-column-density absorber the change in the ionisation parameter goes in the direction of the flux variability but significantly exceeds the expected value: the observed 
Table 5. Spectral fits of the emission/absorption features of PG $2112+059$.

\begin{tabular}{|c|c|c|c|c|c|c|c|c|}
\hline Fit & $\begin{array}{l}\text { Emission } \\
\text { Line model }\end{array}$ & $\begin{array}{c}\text { Energy }^{b} \\
{[\mathrm{keV}]}\end{array}$ & Parameter $^{c}$ & $\begin{array}{c}\text { Norm }^{d} \\
{\left[\mathrm{~cm}^{-2} \mathrm{~s}^{-1}\right]}\end{array}$ & $\begin{array}{c}R(\text { in }) \\
{\left[\mathrm{G} \mathrm{M} \mathrm{c}^{-2}\right]}\end{array}$ & Index & $\begin{array}{c}\text { Inclination }^{e} \\
\text { [degree] }\end{array}$ & $\begin{array}{c}\Xi^{g} \\
{\left[\mathrm{erg} \mathrm{cm} \mathrm{s}^{-1} \text { ] }\right.}\end{array}$ \\
\hline \multicolumn{9}{|c|}{ High state / observation from May 2003} \\
\hline $\begin{array}{l}5 \\
6 \\
7\end{array}$ & $\begin{array}{l}g a \\
b l(r e) \\
b l(r e)\end{array}$ & $6.40^{f}$ & $W=0.0^{f} \mathrm{eV}$ & $\begin{array}{l}1.4_{-1.4}^{+2.5} \times 10^{-6} \\
6.0_{-5.2}^{+3.9} \times 10^{-7} \\
6.4_{-6.3}^{+8.3} \times 10^{-7}\end{array}$ & $\begin{array}{l}5.5_{-4.3}^{+95} \\
1.235^{f}\end{array}$ & $\begin{array}{l}10.0_{-6.4}^{+0.0} \\
2.9_{-2.9}^{+7.1^{+}}\end{array}$ & $\begin{array}{l}34_{+22}^{-32} \\
21_{+69}^{-21}\end{array}$ & $\begin{array}{l}31_{-31}^{+84} \\
31_{-31}^{+59}\end{array}$ \\
\hline \multicolumn{9}{|c|}{ Deep minimum state / observation from November 2005} \\
\hline $\begin{array}{l}12 \\
14^{\dagger} \\
15^{\dagger} \\
17 \\
18 \\
19\end{array}$ & $\begin{array}{l}g a \\
e d \\
n o \\
l a \\
b l(r e) \\
b l(r e)\end{array}$ & $\begin{array}{l}6.40^{f} \\
7.8_{-0.3}^{+0.5} \\
8.2_{-0.6}^{+0.4} \\
4.0_{-0.1}^{+0.1}\end{array}$ & $\begin{array}{l}W=0.0^{f} \mathrm{eV} \\
\tau=1.3_{-0.5}^{+0.6} \\
c f=0.45_{-0.15}^{+0.13} \\
\left(E W=26.1_{-2.8}^{+2.6} \mathrm{keV}\right)\end{array}$ & $\begin{array}{l}7.1_{-0.8}^{+0.7} \times 10^{-6} \\
5.7_{-2.5}^{+4.7} \times 10^{-7} \\
4.1_{-1.3}^{+5.4} \times 10^{-7}\end{array}$ & $\begin{array}{l}1.6_{-0.2}^{+0.3} \\
1.9_{-0.5}^{+0.5} \\
1.235^{f}\end{array}$ & $\begin{array}{l}2.8_{-0.4}^{+0.5} \\
6.2_{-3.9}^{+3.8} \\
2.9_{-0.2}^{+0.3}\end{array}$ & $\begin{array}{c}86.6_{-0.3}^{+1.8} \\
58_{-21}^{+11} \\
39_{-7}^{+9}\end{array}$ & $\begin{array}{l}30_{-30}^{+11} \\
34_{-34}^{+11}\end{array}$ \\
\hline \multicolumn{9}{|c|}{ Both states $^{(h)}$ (high / deep minimum) } \\
\hline 23 & $b l(r e)$ & & & $\begin{array}{l}6.3_{-2.2}^{+3.6} \times 10^{-7} \\
3.7_{-0.8}^{+1.2} \times 10^{-7}\end{array}$ & $2.7_{+0.9}^{-0.6}$ & $3.2_{-0.4}^{+3.1}$ & $40_{-7}^{+14}$ & $37_{-4}^{+9}$ \\
\hline 24 & $b l(r e)$ & & & $\begin{array}{l}6.4_{-2.1}^{+3.8} \times 10^{-7} \\
3.6_{-07}^{+1.2} \times 10^{-7}\end{array}$ & $1.235^{f}$ & $2.8_{-0.2}^{+0.2}$ & $38_{-7}^{+6}$ & $36_{-4}^{+8}$ \\
\hline 25 & $\begin{array}{l}b l(r e) \\
b l(r e)\end{array}$ & & & $\begin{array}{l}5.8_{-5.4}^{+5.0} \times 10^{-7} \\
3.6_{-0.9}^{+1: 2} \times 10^{-7}\end{array}$ & $1.235^{f}$ & $2.8_{-0.2}^{+0.2}$ & $38_{-7}^{+6}$ & $\begin{array}{c}40_{-40}^{+151} \\
37_{-4}^{+8}\end{array}$ \\
\hline
\end{tabular}

The corresponding continuum fits are provided in Table 4. Fits labeled with $\dagger$ were performed over the $2-10 \mathrm{keV}$ energy range for MOS and the 2-12 keV energy range for pn. ${ }^{a}$ The following models are used: $e d$ : absorption edge at the redshift of the source (XSPEC: zedge), no: notch line absorption (XSPEC: notch), ga: Gaussian line at the redshift of the source (XSPEC: zgauss), la: relativistic disk line after Laor (XSPEC: laor, Laor 1997), maximal outer radius $R_{\text {out }}=400, b l$ : relativistic blurring from an accretion disk around a rotating black hole (XSPEC: kdblur, modified from laor model by Fabian and Johnstone), maximal outer radius $R_{\text {out }}=400$ and iron abundance to iron/solar $=3$, re: reflection by a constant density illuminated atmosphere (XSPEC: Reflion, Ross \& Fabian 2005). Parameters: ${ }^{b}$ energy of line in rest frame of quasar, except for fit $17 ;{ }^{c}$ open parameter; ${ }^{d}$ photon flux; ${ }^{e}$ inclination angle; ${ }^{f}$ fixed; ${ }^{g}$ ionisation parameter. In all cases, where both states are fitted simultaneously, the best estimated parameters are provided in two lines: the first line provides the parameters for the high state and the second line for the deep minimum state. In the second line, only parameters are given which were free to vary with respect to the high state (first line).

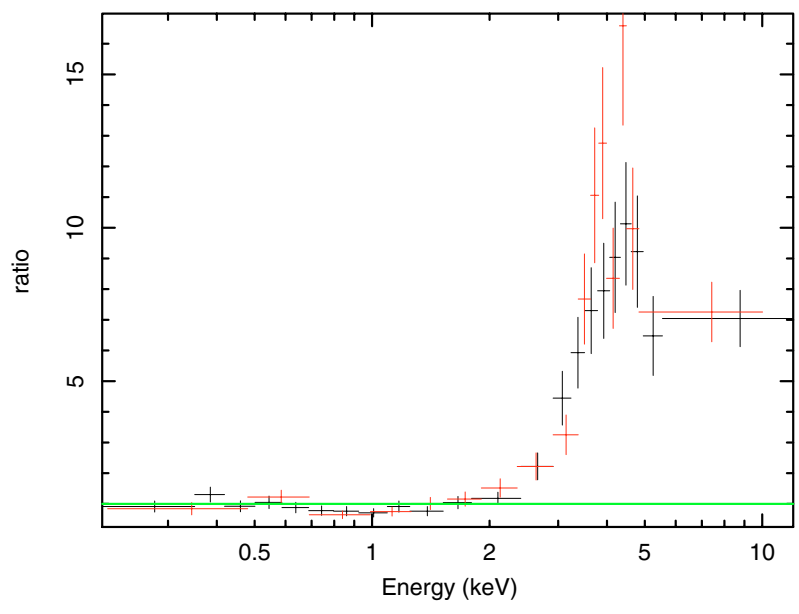

Fig. 1. The ratio between the EPIC data of the deep minimum state of PG $2112+059$ and a hypothetical canonical power law spectra with a photon index of $\Gamma=2$ behind Galactic absorption is shown. The pn data points are given in black and the MOS data points are printed in red, respectively.

ratio of the ionisation parameters is 55 in contrast with a 2.6 flux ratio as expected.

In all three discussed complex absorption scenarios the ionization of the absorber does not follow the flux variation as expected. This inconsistency is not an artefact of fitting both states simultaneously (fit 20, fit 21 and fit 22). The inconsistency is present even when the two states are fitted separately with different column densities (fit 2 and fit 9, fit 3 and fit 10, fit 4 and

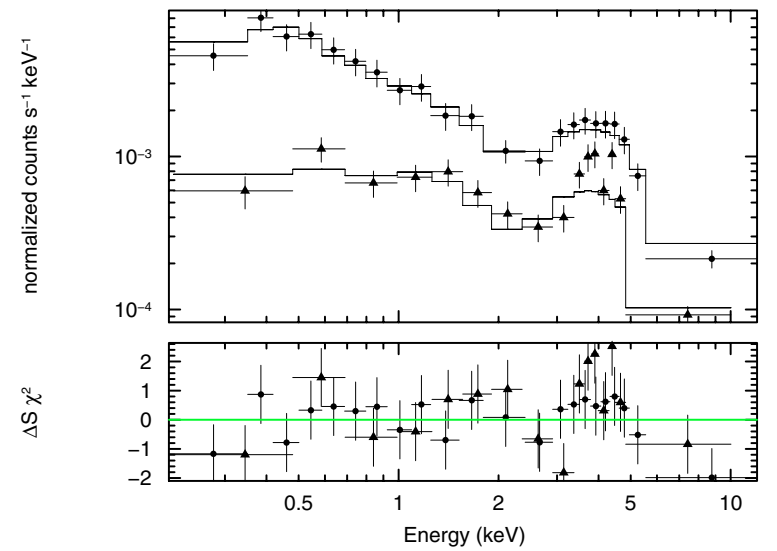

Fig. 2. The two (MOS and pn) EPIC spectra of the deep minimum state of PG $2112+059$ are shown in comparison to a spectral model that consists of a continuum power law behind two layers of absorbing material (Table 4, fit 10). The upper spectrum was taken with the EPIC pn camera and the lower one with the MOS cameras. The model is not able to describe the spectra well at energies above $3 \mathrm{keV}$ as there are systematic residua visible in the lower panel.

fit 11) although errors are clearly larger, which prevents firm conclusions.

Broad absorption line quasars, like PG 2112+059, are characterized by outflows with significant velocities. Based on HST observations Gallagher et al. (2004) determined the outflow velocities of four variable UV absorbing features in the spectrum of PG $2112+059$ to be $9900,13500,16500$ and $20100 \mathrm{~km} \mathrm{~s}^{-1}$. 
Therefore we considered the possibility that the X-ray absorbers are also present in the form of powerful winds with velocity gradients as represented in the "SWIND" model (Gierliński \& Done 2006; Gierliński \& Done 2004). The model allows a fair description of the high state of PG 2112+059 (fit 13) but is not able to provide a valid description of the deep minimum state $\left(\chi^{2}=86.3\right.$, d.o.f. $\left.=32\right)$ and as such the scenario was not considered further for the entire energy band.

\subsection{Lines, bumps and reflection models}

We chose the absorption scenario that is composed of a partial covering neutral absorber and a total covering ionized absorber (i.e. fit 10) to determine upper limits for the presence of a narrow neutral iron emission line. To do this we added a Gaussian line profile to the model with the position and the width fixed to $6.4 \mathrm{keV}$ and $0.0 \mathrm{eV}$, respectively, leaving the normalization as a free parameter. For the high state of the source (fit 5) inclusion of a Gaussian line reduces the value of $\chi^{2}$ by $\Delta \chi^{2}=0.9$ (3- $\sigma$ upper-limit to $E W_{3 \sigma} \leq 559 \mathrm{eV}$ ). For the deep minimum state (fit 12) the value of the $\chi^{2}$ decreases by $\Delta \chi^{2}=6.6$, corresponding to a 2.6- $\sigma$ detection of the line with an equivalent width of $E W=160_{-110}^{+100} \mathrm{eV}$. The corresponding 3- $\sigma$ upper-limit is $E W_{3 \sigma} \leq 354 \mathrm{eV}$. As the fit without a Gaussian line shows systematic residua over the entire bump region we cannot consider the improvement in $\chi^{2}$ as an indication of the presence of a narrow neutral $6.4 \mathrm{keV}$ iron line.

Although the discussed absorption scenarios provide statistically acceptable descriptions of the spectra, the models fail to provide a good description of the bump observed during the deep minimum state as there are always residua left, compare Fig. 2. These residua are too large and too systematic in character to be explained with current calibration uncertainties.

In order to study the nature of the $3-6 \mathrm{keV}$ bump of the deep minimum state in detail we restricted the spectral analysis to the $2-10 \mathrm{keV}$ energy range of the spectra. As expected the spectra can be described with a power law continuum and a neutral absorber that covers the continuum source partially. The powerlaw index of the continuum is estimated to be $\Gamma=3.54_{-0.57}^{+0.43}$. As changes of the covering factor essentially only impact the low energy region, the power-law index is mainly determined by the spectral shape at energies $>6 \mathrm{keV}$ in the rest frame of the source. But a power-law index of this value is rather unusual for AGN in this energy region (Piconcelli et al. 2005). We therefore modelled the spectra with a partially covering neutral absorber with an additional absorption edge assuming a power law continuum with a photon index fixed to the canonical $\Gamma=2.0$ (fit 14). The estimate for the energy of the absorption edge, $E=7.78_{-0.31}^{+0.78} \mathrm{keV}$, is above the value of neutral iron. However, at the 3- $\sigma$ level, absorption by neutral iron cannot be excluded $\left(E_{3 \sigma}=6.92 \mathrm{keV}\right)$. In addition we tested a model in which the edge was replaced by an absorption feature with the shape of a notch (fit 15). The lower border of the notch was estimated to be $E_{\text {low }}=7.59_{-0.87}^{+0.51} \mathrm{keV}$, where the values are provided in the rest frame to the quasar. Again an absorption by neutral iron cannot be excluded $\left(E_{\text {low } / 3 \sigma}=6.40 \mathrm{keV}\right)$. To summarize, the analysis of the high energy range of the spectra shows that the residua can be reduced by either assuming an uncommon steep continuum at energies $>6 \mathrm{keV}$ or an additional, third, absorption feature. If the absorber is identified with iron then ionised material would be preferred, but neutral iron cannot be excluded.

As an absorbing edge as well as a notch are rather simple and likely unrealistic models we tried two, more sophisticated absorption scenarios to test if the bump can be interpreted as an artefact of an insufficient description of absorption. We use the deep minimum spectra limited to the $2-10 \mathrm{keV}$ energy range and explored models that allow one to account for winds with velocity dispersions. First we tried the "SWIND" (Gierliński \& Done 2006; Gierliński \& Done 2004) model. A "SWIND"-absorbed power law continuum is able to describe the $2-10 \mathrm{keV}$ spectra from a statistical point of view (fit 16), although the obtained $\chi^{2}$-value is rather high, $\chi^{2}=21.12$ with respect to d.o.f. $=13$. But most important, the best estimate of the photon index of the power law continuum, $\Gamma=3.70_{-0.64}^{+1.59}$ is too high for the modelled energy range. An attempt to describe the spectra with a "SWIND"-absorber and the photon index of the continuum fixed to the canonical value, $\Gamma_{\text {canonical }}=2.0$. failed $\left(\chi^{2}=39.7\right.$ for d.o.f. $=14)$. The second complex absorption model that we tried is XSTAR (Kallman 2007). Within the statistical analysis program, XSPEC, the XSTAR model is provided as pre-calculated tables as well as in analytical form. Currently, the analytical from is not fully tested and requires a substantial amount of computing power, but offers the advantage that more parameters are allowed to be varied than in the tables. Our attempts to model the 0.2-10 keV spectra of the deep minimum state with the XSTAR table models failed. Statistically acceptable fits could only be obtained at the expense of unrealistically high photon indices, e.g. $\Gamma=6.77_{-0.63}^{+0.55}$ for $z=0.457\left(\chi^{2}=20.4\right.$ for d.o.f. $\left.=15\right)$ or $\Gamma=4.82_{-0.31}^{+0.35}$ for $z=0.457_{-0.457}^{+0.0}\left(\chi^{2}=20.5\right.$ for d.o.f. $\left.=14\right)$. Attempts to model the spectra with a canonical photon index, $\Gamma_{\text {canonical }}=2.0$, were statistically unacceptable $\left(\chi^{2}=37.4\right.$ for d.o.f. $=15$ for $z=0.457_{-0.457}^{+0.0}$ and $\chi^{2}=37.4$ for d.o.f. $=16$ for $z=0.457)$. Trials with the analytical XSTAR model confirmed the results of the table models. Either the fits are statistically unacceptable or unrealistic parameters were estimated. The possibility to vary the iron abundance could not be utilized to improve the situation as unrealistically high values are estimated. Again, we always cross-checked the results by trying fits with parameters fixed to the canonical values, e.g. $\Gamma_{\text {canonical }}=2.0$. and iron/solar $=3$, and the models were statistically unacceptable.

Since absorption does not seem to be the best possible interpretation of the 3-6 keV bump observed during the deep minimum state, we tried to model the spectra (without limiting to the hard emission) with a power law continuum absorbed by ionised material plus (1) an additional Gaussian line or (2) a relativistic broadened emission line emitted by an accretion disk around a Kerr black hole (Laor 1991). The parameter estimates result in both cases in very high column densities $\left(N_{\mathrm{H}}=6.6_{-1.9}^{+2.9} \times 10^{23} \mathrm{~cm}^{-2}\right.$ and $\left.N_{\mathrm{H}}=9.4_{-4.0}^{+0.6} \times 10^{23} \mathrm{~cm}^{-2}\right)$ for the ionised absorber in combination with very high ionisation parameters $\left(\xi=670_{-140}^{+310} \mathrm{erg} \mathrm{cm} \mathrm{s}^{-1}\right.$ and $\left.\xi=884_{-390}^{+750} \mathrm{erg} \mathrm{cm} \mathrm{s}^{-1}\right)$ and a very flat (Piconcelli et al. 2005) power law continuum $\left(\Gamma=1.37_{-0.10}^{+0.11}\right.$ and $\left.\Gamma=1.30_{-0.06}^{+0.08}\right)$. Given these parameter estimates, in both cases the warm absorber is involved in at least part of the 3-6 keV bump emission, whereas the continuum is unusually flat.

Next we tried to describe the continuum with a power law absorbed by neutral material. In the case of a description of the bump through a Gaussian line, the power law continuum is estimated to be unusually steep, $\Gamma=2.67_{-0.51}^{+0.37}$ in comparison with other quasars (Piconcelli et al. 2005). The steep continuum leads to a severe underestimation of the continuum contribution for the region of the bump which consequently causes an unrealistically wide equivalent width. Therefore the scenario was not considered further. The situation looks better if the bump is modelled with a relativistic broadened emission line (fit 17), where we fixed the outer radius of the disk. The best estimated power law index, $\Gamma=1.95_{-0.20}^{+0.22}$, is in the range expected for quasars but we 


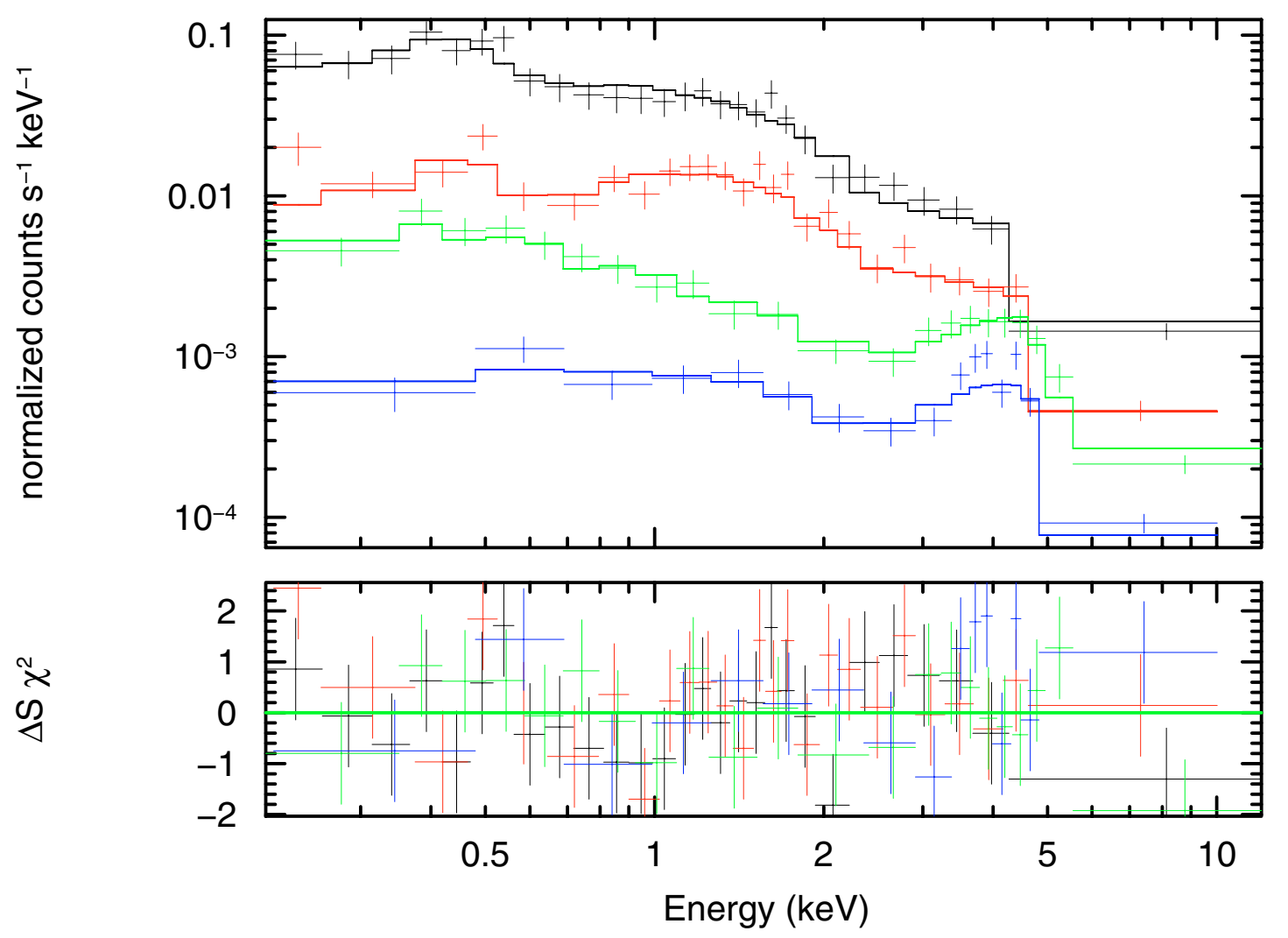

Fig. 3. The EPIC spectra of both observations plotted in comparison to a model consisting of a continuum power law and a relativistically blurred $\mathrm{X}$-ray ionised reflection component that originates in an accretion disk near to a supermassive black hole. Both components are seen through a warm absorber (Table 4, fit 23). The ionisation parameter of the warm absorber and the normalization of the continuum components were free to vary. The deep minimum state is dominated by the reflection component while the power law component vanishes.

formally obtain $E W=26.1_{-2.8}^{+2.6} \mathrm{keV}$. The model to data comparison shows systematic residua and the fit does not appear to be statistically very satisfying $\left(\chi^{2}=50.7\right.$, d.o.f. $\left.=30\right)$. Such a value of the equivalent width indicates that the bump is by far dominated by the line emission for energies above $3 \mathrm{keV}$. From a physical viewpoint the obtained equivalent width shows that the neutral absorber plus an emission line model cannot account for the measured spectrum and that especially the reflection continuum must be considered accordingly.

Consequently we tested whether the deep minimum state is able to be interpreted by X-ray ionised reflection on the accretion disk. We assumed a continuum spectrum composed of a power law and an ionised-reflection model (Ross \& Fabian 2005) that is blurred with a Laor line profile (Ross \& Fabian 2005; Crummy et al. 2005). We model the data with this continuum and an ionised absorber in the line of sight. We restricted the free parameters of the warm absorber by assuming a temperature of $T=3 \times 10^{5} \mathrm{~K}$ and solar iron abundance. In addition we assumed that the power law component and the reflection model have the same, but free, index. This model allows an excellent statistical description of the data although the parameters of the relativistic blurring cannot be well constrained. The best parameter estimates either provide a very low value for the outer radius of the emission region of the accretion disk in combination with very high iron abundance relative to the solar abundance or a very steep index for the power law emissivity. We therefore fixed in all models the outer radius of the disk emission region to its maximal radius $R_{\text {out }}=400$ and the iron abundance to iron/solar $=3$. Modelling of the spectra with either the inner radius of the emission region as a free parameter (fit 18) or fixing the inner radius to its minimal possible value $R_{\text {in }}=1.235$ (fit 19) result in acceptable descriptions $\left(\chi^{2}=29.9\right.$ for d.o.f. $=29$ and $\chi^{2}=35.7$ for d.o.f. $=30)$ of the data obtained during the deep minimum state of the source. For completeness we verified that the described model is also able to describe the high state of the source (fit 6 and fit 7).

Finally we tested whether a model that consists of a power law and a relativistically blurred ionised reflection behind an ionised absorber can explain both observations of PG 2112+059 in a consistent and simple way. To do this we fitted both data sets simultaneously. Again we reduced the number of free parameters by fixing parameters as specified above. In addition we coupled the large majority of the parameters such that the fit was forced to find an estimate describing both observations. Between the two observations only three parameters were allowed to vary: the normalizations of the two continuum components and the ionisation parameter of the warm absorber. The model provides an excellent description of the data; no systematic residua are left over if the model is compared with the data (Fig. 3). Similar to the modulations described in the previous paragraph, it was necessary to constrain the relativistic blurring by fixing the iron abundance to three times the solar value and the outer radius of the disk emission region to $R_{\text {out }}=400$. Both fits, with free inner radius (fit 23) and with the inner radius fixed to the minimal possible inner radius (fit 24) provide statistically excellent descriptions of the data $\left(\chi^{2}=79.9\right.$ for d.o.f. $=80$ and $\chi^{2}=83.9$ for d.o.f. $=81)$ without showing systematic residua.

The limited statistics required a freezing of two parameters that describe the ionised-reflection model blurred with a Laor line profile and therefore the estimated parameters cannot be 
interpreted in a simple way. Nevertheless the models provide a plausible description of the data which is in agreement with general expectations and measurements.

\section{Discussion}

A simple model, consisting of a power law continuum behind a layer of absorbing material, fails to describe both states of PG $2112+059$ in a consistent way. The upper limits obtained for a possible narrow iron fluorescence emission line are not decisive for an interpretation in the context of absorption. This is because Seyfert 2 AGNs with similar absorbing column densities show higher as well as lower equivalent widths (Guainazzi et al. 2005).

The spectra can be described with a power law continuum absorbed by two different absorbers, one ionised and the other only partially covering the source. Here two geometrical scenarios are possible: either the two absorbers are located behind each other or the two absorbers only partially cover the continuum source. A description of the spectra in the two states requires a change of the covering fraction of the neutral material as well as a change of the ionisation parameter. Therefore, absorbing material has to move with respect to the continuum source and our line of sight.

Although the discussed absorption scenarios provide statistically acceptable descriptions of the spectra there are several arguments against this interpretation:

- The 3-6 keV excess emission above the continuum powerlaw observed during the deep minimum state is not well described as there are always systematic residua left, - compare Fig. 2.

- The residua at energies $>6 \mathrm{keV}$ in the rest frame of the source can be reduced if the continuum emission is described with a power law with $\Gamma=3.5$. However, such a steep continuum has no parallel in other AGNs (Piconcelli et al. 2005) and as such is very unlikely to explain the observation.

- Another possibility would be to explain the behaviour at energies $>6 \mathrm{keV}$ with absorption similar to that discussed by Boller et al. (2002) for $1 \mathrm{H}$ 0707-49. A more general recent discussion of the topic can be found in Boller (2006). However, with this assumption we introduce a further, third, absorption component.

- But most important is that in all scenarios the ionisation parameter of the deep minimum state is estimated to be significantly higher than the ionisation parameter found for the high state, in sharp contrast to the expectation that a higher source flux should cause a higher ionisation state.

All together the arguments are against an interpretation of the spectra uniquely in terms of absorption.

In view of these we tested models that are composed of a power law continuum and an X-ray ionised reflection on the accretion disk. These models lead to statistically excellent descriptions of the data and especially allow us to describe both states in a consistent way. There are two issues requiring further discussion: the ionisation parameter of the warm absorber and the disappearance of the power law continuum component during the deep minimum state.

An interpretation in the sense of reflection on an accretion disk leads to values for the ionisation parameters of the absorber which are much more consistent with values commonly found for AGNs. But most important is the fact that the change of the ionisation parameter is consistent with the variation of the flux. Comparing the continuum flux normalization measured for the high state of the source with the $90 \%$ upper limit of the continuum normalization obtained for the deep minimum state (fit 24) we expect a drop of the ionisation parameter by a factor of 100 . From the measured ionisation parameters we obtain a ratio of $27_{-23}^{+420}$. The change of the ionisation parameters therefore goes qualitatively in the expected direction. Even quantitatively the measured ratio of the ionisation parameter is in agreement with the value expected from the change of the continuum flux. For comparison none of the absorption scenarios was able to provide only qualitatively a consistent picture. As the larger reflection fraction during the deep minimum may imply a higher ionization of the reflector we modelled both states simultaneously with the ionization of the reflector as free parameter (fit 25). Unfortunately, the event statistics obtained are insufficient to measure any real difference. Finally we verified that the best estimate for the ionisation parameter of the deep minimum is a global minimum by stepping the parameter through the allowed range.

The joint modelling of both spectral states of the source spectra leads to a description of the deep minimum state in which the power law continuum component completely vanishes, i.e. the whole observed spectrum is interpreted as emission from X-ray ionised reflection on an accretion disk. Although it is unexpected to have AGN spectra without the paradigmatic power law component, we note that Miniutti \& Fabian (2004) predicted exactly this situation.

In order to explain observed uncorrelated variability between direct continuum and reflection components including the iron line, Miniutti \& Fabian (2004) explored a light-bending model. They assumed a primary source of X-rays located close to a central, maximal rotating Kerr black hole and illuminating both the observer at infinity and the accretion disk. The authors showed that even in the case of intrinsic constant luminosity the observed flux can vary by more that an order of magnitude as the height of the primary source above the accretion disks varies due to strong light bending. Miniutti and Fabian identified three different regimes (named regime I, II and III) according to the correlation between the reflection-dominated component and the direct continuum.

The deep minimum state of PG $2112+059$ can be interpreted as an observation in the regime I in which the observed direct continuum is very low. In this regime the primary source is at low height such that the primary source emission suffers from strong light bending that dramatically reduces the power law continuum at infinity (Miniutti \& Fabian 2004). In addition Miniutti and Fabian predicted for regime I a very broad iron line emission profile without any prominent "horn"-feature (compare Fig. 4 in Miniutti \& Fabian 2004). The observed 3-6 keV "bump" during the deep minimum state is qualitatively in agreement with the prediction.

There are several other AGNs for which absorption versus relativistically blurred X-ray ionised reflection on an accretion disk is discussed. Examples are 1H 0707-495 (Boller et al. 2002; Fabian et al. 2002; Tanaka et al. 2004; Fabian et al. 2004) or 1H 0419-577 (Fabian et al. 2005; Pounds et al. 2004a,b). But in all these cases an absorption scenario is much more plausible than in the case of PG $2112+059$. A deep minimum state with the complete disappearance of the continuum power law was reported for only three other AGNs: NGC 4051 (Uttley et al. 1999), NGC 2992 (Gilli et al. 2000) and NGC 1365 (Risaliti et al. 2007). In all these cases the authors report narrow iron line emission with equivalent widths of $>0.7 \mathrm{keV}$, i.e. values which are above the $3-\sigma$ upper-limits obtained here for PG $2112+059$. Consequently in all cases the authors explain the deep minimum state with reflection on 
material distant to the supermassive black hole (e.g. broadline region in the case of NGC 1365). The appearance of the deep minimum is either explained by absorbing material moving into the line of sight (NGC 1365) or a break down of the accretion process and primary X-ray emission (NGC 2992 and NGC 4051). Therefore, PG $2112+059$ appears to be the best example of an AGN where the effect of light-bending close to the central black hole has been detected so far.

A better statistical basis is required to finally decide on the nature of the X-ray emission of PG $2112+059$. To achieve this objective we have successfully applied for a $220 \mathrm{ks}$ long observation of PG $2112+059$ with XMM-Newton which is planned to be only performed if the quasar is found in the deep minimum state. If we can obtain such a spectrum and if the current interpretation (a relativistially blurred ionised X-ray reflection emitted from an accretion disk with the power law continuum suppressed, or even not present, due to light blending) holds, then there are several quite remarkable consequences:

- The spectra and the associated variability pattern will allow us to test the predictions of the light-bending model (Miniutti \& Fabian 2004). Such data will allow us to compare the emission by an accretion disk near the supermassive black hole with theoretical predictions with unprecedented accuracy.

- The question arises weather the weakness of other X-ray weak quasars is caused by light bending, too. A hint might be provided by PG $1535+547$, which shows a very similar spectral and temporal behaviour (Ballo et al. 2007). The modelling of several XMM-Newton spectra of X-ray weak quasars lead to warm absorbers with very high ionisation parameters (e.g. Schartel et al. 2005, for PG 1535+547 and PG 1001+054; Brinkmann et al. 2004, for PG 1411+442 and Piconcelli et al. 2004, for Mrk 304; Boller et al. 2002; Fabian et al. 2002; Tanaka et al. 2004; and Fabian et al. 2004, for 1H 0707-495 ). Similar to PG 2112+059, in these cases the high ionisation parameters might be a hint of a more complex spectral scenario and especially of X-ray ionised reflection on accretion disks. The statement that absorption is the primary cause of the weakness of (soft) X-ray weak quasars (Brandt et al. 2000) might be challenged. In addition the question about the nature of quasar types that are closely related to X-ray weak quasars, like BAL quasars (Brandt et al. 2000), would be raised, too.

- Many observations of quasars in the X-ray region aim to find features that originate near to the central supermassive black hole. Due to the still very limited effective area of the current generation of X-ray telescopes a strong selection for X-ray bright sources usually is required to keep the exposure time reasonably long. But this might be a bias, as the selection favours a high flux of the continuum power law with respect to the relativistic blurred X-ray ionised reflection from the accretion disk, which has a very small equivalent width and is hard to detect.

- Further spectra in combination with their temporal characteristics might even open the possibility of testing the general theory of relativity in the strong field region with the current generation of X-ray satellites, as suggest at the workshop "Rethinking gravity" (Hogan 2007).

\section{Conclusions}

1. In November 2005 PG $2112+059$ was in a deep minimum state where the flux was lower by a factor of 10 in comparison to the May 2003 data.
2. An interpretation of the spectral changes in the context of an absorption scenario, although statistically acceptable, is unsatisfying: the modelling with a neutral and an ionised absorber still leaves systematic residua, results in high column densities and very high ionisation parameters. Especially, the ionisation parameters found for the deep minimum are higher than the ionisation parameters found for the high state, implying either the presence of physically different absorbers or a significant movement of the absorbers relative to the X-ray sources.

3. An interpretation in the context of relativistically blurred $\mathrm{X}$-ray ionised reflection on an accretion disk near the supermassive black hole behind a warm absorber provides a plausible scenario for the spectral changes. In this case the deep minimum state shows no continuum component which can be explained by light bending (Miniutti \& Fabian 2004). In this scenario we obtain ionisation parameter values that are typical of AGNs, and especially find for the deep minimum state lower ionisation parameters than for the high state, as expected for a static absorber.

4. Should further observations confirm the light-bending scenario for PG $2112+059$ then the source will offer unique possibilities to specifically test the light-bending model and the emission of an accretion disk near the supermassive back hole in general.

5. The finding for PG $2112+059$ challenges the suggestion that absorption is the primary cause of the weakness of X-ray weak quasars.

Acknowledgements. Based on observations obtained with XMM-Newton, an ESA science mission with instruments and contributions directly funded by ESA Member States and NASA.

We thank the referee, Dr. Giovanni Miniutti, for the many useful comments.

\section{References}

Arnaud, K., Dorman, B., \& Gordon, D. 2006, XSPEC: An X-Ray Spectral Fitting Package, User's Guide for version 12.3.0

http://heasarc.nasa.gov/docs/xanadu/xspec/XspecManual.pdf Avni, Y. 1976, ApJ, 210, 642

Ballo, L., et al. 2007 in preparation

Brandt, W. N., Laor, A., \& Wills, B. J. 2000, ApJ, 528, 637

Boller, T. 2006, AN, 327, 1071

Boller, T., Fabian, A. C., Sunyaev, R., et al. 2002, MNRAS, 329, 1

Boroson, T. A., \& Green, R. F. 1992, ApJS, 80, 109

Brinkman, A. C., Behar, E., Güdel, M., et al. 2001, A\&A, 365, L324

Brinkmann, W., Papadakis, I. E., \& Ferrero, E. 2004, A\&A, 414, 107

Crummy, J., Fabian, A. C., Brandt, W. N., et al. 2005, MNRAS, 361, 1197

Elvis, M., \& Fabbiano, G. 1984, ApJ, 280, 91

Fabian, A. C., Ballantyne, D. R., Merloni, A., et al. 2002, MNRAS, 331, L35

Fabian, A. C., Miniutti, G., Gallo, L., et al. 2004, MNRAS, 353, 1071

Fabian, A. C., Miniutti, G., Iwasawa, K., \& Ross, R. R. 2005, MNRAS, 361, 795

Gallagher, S. C., Brandt, W. N., Laor, A., et al. 2001, ApJ, 546, 795

Gallagher, S. C., Brandt, W. N., Wills, B. J., et al. 2004, ApJ, 603, 425

Gierliński, M., \& Done, C. 2004, MNRAS, 349, 7

Gierliński, M., \& Done, C. 2006, MNRAS, 371, 16

Gilli, R., Maiolino, R., Marconi, A., et al. 2000, A\&A, 355, 485

Guainazzi, M., Matt, G., \& Perola, G. C. 2005, A\&A, 444, 119

Hogan, J. 2007, Nature, 445, 468

Jansen, F., Lumb, D., Altieri, B., et al. 2001, A\&A, 365, L1

Jannuzi, B. T., Bahcall, J. N., Bergeron, J., et al. 1998, ApJSS, 118, 1

Kallman, T. R. 2007, XSTAR, A Spectral Analysis Tool, Version 2.1kn7, heasarc.gsfc.nasa.gov/xstar/xstar.html

Kendall, M. G., \& Stuart, A. 1973, The advanced Theory of Statistics (New York: Hafner), 2, 97

Kirsch, M. 2006, EPIC status of calibration and data analysis, Issue 2.5, XMMSOC-CAL-TN-0018, http://xmm.esac.esa.int/docs/documents/ CAL-TN-0018.pdf

Laor, A. 1991, ApJ, 376, 90

Laor, A., Fiore, F., Elvis, M., et al. 1997, ApJ, 477, 93 
Lockman, F. J., \& Savage, B. D. 1995, ApJS, 97, 1-47

Loiseau, N. 2006, User's Guide to the XMM-Newton Science Analysis System, Issue 4.0 ,

http://xmm.vilspa.esa.es/external/xmm_user_support/ documentation/sas_usg/USG/USG.html

Mason, K. O., Breeveld, A., Much, R., et al. 2001, A\&A, 365, 36

Miniutti, G., \& Fabian, A. C. 2004, MNRAS, 1993, 349, 1435

Piconcelli, E., Jimenez-Bailón, E., Guainazzi, M., et al. 2004, MNRAS, 351, 161

Piconcelli, E., Jimenez-Bailón, E., Guainazzi, M., et al. 2005, A\&A, 432, 15

Pounds, K. A., Reeves, J. N., Page, K. L., \& O'Brien, P. T. 2004a, ApJ, 605, 670

Pounds, K. A., Reeves, J. N., Page, K. L., \& O’Brien, P. T. 2004b, ApJ, 616, 696

Risaliti, G., Elvis, M., Fabbiano, G., et al. 2007, ApJ, 659, 111
Ross, R. R., \& Fabian, A. C. 2005, MNRAS, 358, 211

Schartel, N., Rodríguez-Pascual, P. M., Santos-Lleó, M., et al. 2005, A\&A, 433, 455

Schmidt, M., \& Green, R. F. 1983, ApJ, 269, 353

Strüder, L., Briel, U., Dennerl, K., et al. 2001, A\&A, 365, L18

Tanaka, Y., Boller, T., Gallo, L., et al. 2004, PASJ, 56, 9

Turner, M. J. L., Abbey, A., Arnaud, M., et al. 2001, A\&A, 365, L27

Uttley, P., McHardy, I. M., Papadakis, I. E., et al. 1999, MNRAS, 307, 6

Véron-Cetty, M.-P., \& Véron, P. 200, A Catalogue of Quasars and Active Nuclei, 9th edn, Scientific Report No 19, European Southern Observatory, München

Watson, M. G., Auguéres, J.-L., Ballet, J., et al. 2001, A\&A, 365, L51

Wang, T., Brinkmann, W., \& Bergeron, J. 1996, A\&A, 309, 81 